\title{
Auditory frequency perception adapts rapidly to the immediate past
}

\author{
David Alais • Emily Orchard-Mills • Erik Van der Burg
}

Published online: 19 December 2014

(C) The Psychonomic Society, Inc. 2014

\begin{abstract}
Frequency modulation is critical to human speech. Evidence from psychophysics, neurophysiology, and neuroimaging suggests that there are neuronal populations tuned to this property of speech. Consistent with this, extended exposure to frequency change produces direction specific aftereffects in frequency change detection. We show that this aftereffect occurs extremely rapidly, requiring only a single trial of just $100-\mathrm{ms}$ duration. We demonstrate this using a long, randomized series of frequency sweeps (both upward and downward, by varying amounts) and analyzing intertrial adaptation effects. We show the point of constant frequency is shifted systematically towards the previous trial's sweep direction (i.e., a frequency sweep aftereffect). Furthermore, the perception of glide direction is also independently influenced by the glide presented two trials previously. The aftereffect is frequency tuned, as exposure to a frequency sweep from a set centered on $1,000 \mathrm{~Hz}$ does not influence a subsequent trial drawn from a set centered on $400 \mathrm{~Hz}$. More generally, the rapidity of adaptation suggests the auditory system is constantly adapting and "tuning" itself to the most recent environmental conditions.
\end{abstract}

Keywords Adaptation and Aftereffects $\cdot$ Audition $\cdot$ Hearing

\section{Introduction}

Adaptation has been used for decades in psychophysical research as a method to infer the properties of neurons underlying perception of a given stimulus. Long before neuroimaging and electrophysiology were commonplace, the use of

D. Alais $(\bowtie) \cdot$ E. Orchard-Mills $\cdot$ E. Van der Burg

School of Psychology, University of Sydney, Griffith Taylor

Building A19, Sydney, NSW 2006, Australia

e-mail: david.alais@sydney.edu.au extended exposure to stimuli and their consequent perceptual aftereffects served as the "psychologist's micro-electrode" (Frisby, 1980). In vision research, this approach was used to investigate neurons tuned to colour, motion, spatial frequency and orientation, to name but a few stimulus dimensions (for reviews see Clifford et al., 2007; Webster, 2011). Similarly, adaptation has been extensively employed in auditory (Carlile, Hyams, \& Delaney, 2001; Kay \& Matthews, 1972; Phillips \& Hall, 2005), somatosensory (Hahn, 1966; Hollins, Sliman, \& Washburn, 2001; Miyazaki, Yamamoto, Uchida, \& Kitazawa, 2006), and olfactory research (Dalton, 2000; Pryor, Steinmetz, \& Stone, 1970; Steinmetz, Pryor, \& Stone, 1970). Despite its widespread use, there are some limitations to this paradigm. Traditionally, adaptation studies separate exposure and testing into two separate phases, with participants monitoring the adapting stimulus for an extended period after which a test trial is presented. A number of test trials are normally needed to obtain an accurate measure of the aftereffect and so the adaptation period (or sometimes a shorter "top-up" adaptation) must be repeated. This approach is not only very timeconsuming, but also highly inefficient as no useful information is gathered during lengthy periods of adaptation.

Two recent multisensory studies have adopted an alternative approach based on intertrial analyses and shown that adaptation can be induced and tested extremely rapidly (Van der Burg, Alais, \& Cass, 2013; Wozny \& Shams, 2011). In this approach, stimuli are presented in a series of rapid trials with each briefly presenting the stimulus at a level randomly drawn from a range sufficient to measure a threshold. After each trial, participants make a binary forced-choice response to a simple question about the stimulus (e.g., Was the line vertical?; Was the sound intensity constant?). When the trial series is finished, the response to each level of the stimulus on a given trial is binned based on the stimulus level presented in the previous trial. In effect, each trial is both the adapted stimulus from the previous trial and serves as the adapting 
stimulus for the subsequent trial. The key feature of this approach is that there is no division between adaptation and test phases. This improves efficiency as no time is lost in timeconsuming adaptation periods and every trial provides data. Moreover, as recent multisensory studies of intertrial adaptation have shown in both the spatial (Wozny \& Shams, 2011) and temporal (Harvey, Van der Burg, \& Alais, 2014; Van der Burg et al., 2013; Van der Burg, Orchard-Mills, \& Alais, 2014) domains, adaptation effects are evident after a single brief trial. This challenges the assumption in traditional adaptation studies that prolonged exposure to an adaptor is necessary to produce reliable perceptual aftereffects. It also prompts the question of whether aftereffects can be induced rapidly within a single modality.

In order to investigate this question, we applied the rapid intertrial approach to a well-known auditory aftereffect: adaptation to frequency modulation (Gardner \& Wilson, 1979; Kay \& Matthews, 1972; Regan \& Tansley, 1979). Frequency modulation is particularly important for communication, as rapid frequency changes are a key feature for the perception of speech (Delattre, Cooper, Liberman, \& Gerstman, 1954; Liberman \& Mattingly, 1989; Liberman, Cooper, Shankweiler, \& Studdert-Kennedy, 1967) and define meaning in tonal languages (Zeng et al., 2005). This is not only true for humans, but also animals, including many species of bats who use frequency modulation for echolocation (for a review see Altmann \& Gaese, 2014). Previous adaptation studies have demonstrated that prolonged exposure to auditory stimuli with changing frequency increases the threshold for frequency modulation (Gardner \& Wilson, 1979; Green \& Kay, 1973; Kay \& Matthews, 1972; Kayahara, 2001; Masutomi \& Kashino, 2013; Regan \& Tansley, 1979; Shu, Swindale, \& Cynader, 1993; Tansley \& Suffield, 1983), supporting the existence of neural populations tuned to frequency modulation. Confirming this psychophysical inference, neurophysiological studies have found neuronal populations tuned to frequency-modulation in a direction-selective manner in cats (Mendelson \& Cynader, 1985; Mendelson \& Grasse, 1992; Mendelson, Schreiner, Sutter, \& Grasse, 1993), monkeys (Atencio et al., 2007; L. Liang, Lu, \& Wang, 2002; Tian \& Rauschecker, 2004), guinea pigs (Zhao \& Liang, 1996), and bats (Gordon \& O'Neill, 1998), and in humans using neuroimaging techniques (Pardo \& Sams, 1993; Sams et al., 1991).

As adaptation to frequency modulation is well-established perceptually, it is ideal for examining rapid intertrial adaptation within a unimodal context. In this study, participants were presented with brief $(100 \mathrm{~ms})$ upward or downward frequency glides of different excursions in a completely randomised order and were required to indicate the direction of the glide. Performance was then analysed based on the direction of frequency glide in the previous trial. To preview our results, the perception of frequency modulation is dependent on the direction of the previous trial's frequency glide. These results demonstrate that, consistent with the findings in cross-modal studies, adaptation within a single modality can occur rapidly, without requiring a prolonged exposure period.

\section{Experiment 1}

In Experiment 1, we examined whether adaptation to linear frequency glides occurs rapidly without a prolonged adaptation procedure. On each trial, a linear frequency glide with a fixed center frequency of $1,000 \mathrm{~Hz}$ was presented. The frequency excursion $(\Delta \mathrm{f})$ varied randomly across trials $(0, \pm 1 / 24$, $\pm 1 / 12, \pm 1 / 6, \pm 1 / 3$, or \pm 2 octaves) and participants were asked to judge the direction of the glide (either upward or downward) by making an unspeeded response (Fig. 1a). If adaptation to linear frequency glides occurs rapidly, then we expect that the perceived glide direction on the current trial ( $\mathrm{t}$ ) will be contingent upon the glide direction in the preceding trial $(\mathrm{t}-1)$. By binning all trials into two categories based on the previous trial's glide direction, the aggregate effect of the preceding trial should manifest as a lateral shift in the psychometric function towards the adaptor. For trials preceded by a downward (negative) glide, the function should shift to the left so that the point of subjective constancy (PSC) corresponds to an actual downward glide, and vice versa for a preceding upward glide. Experiment 1 tests these predictions.

\section{Method}

Participants Eight students participated in the experiment (two female; mean age: 24.7 years; range 20-29). All participants received AU $\$ 20$ per hour for their participation, and were naïve as to the purpose of the experiment.

Apparatus The experiment was run in a dimly lit experimental booth and was programmed and controlled in Eprime 2.0 software. Participant's responses were recorded using a Dell wired USB keyboard. Auditory stimuli were presented to participants via Sennheiser HD 380 pro headphones.

Stimuli The auditory stimulus was a linear frequency glide with a fixed center frequency of $1,000 \mathrm{~Hz}$ and a duration of $100 \mathrm{~ms}$ (including onset and offset ramps). The frequency excursion $(\Delta f)$ varied across trials $(0, \pm 1 / 24, \pm 1 / 12, \pm 1 / 6, \pm 1 /$ 3 , and \pm 2 octaves, corresponding to glides sweeping between 986-1015, 972-1029, 944-1060, 891-1123, and 500$2000 \mathrm{~Hz}$ ). The glides were created using Matlab's (The MathWorks) inbuilt chirp function at sampling rate of 44, $100 \mathrm{~Hz}$ and resolution of 16 bits. A cosine ramp of $20 \mathrm{~ms}$ was applied to the glide's onset and offset. 
A) Fixed frequency $(1000 \mathrm{~Hz})$

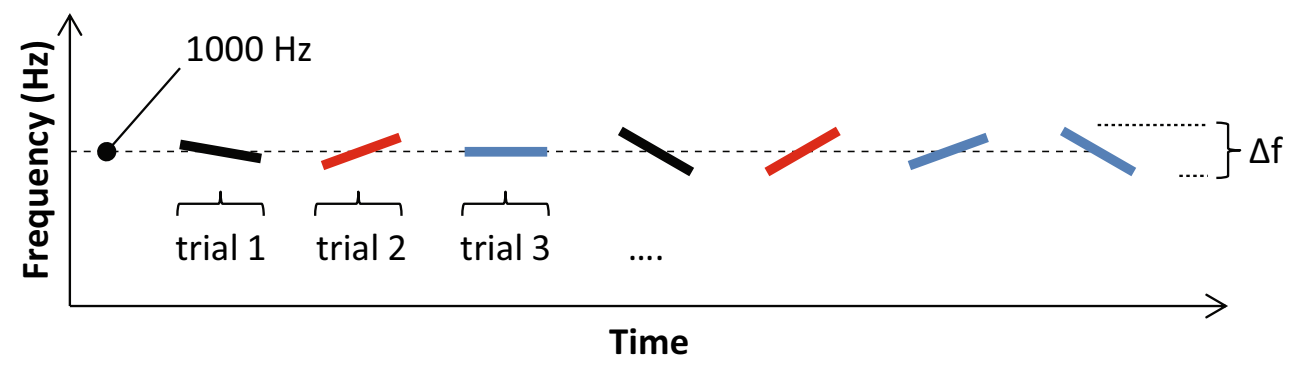

B) Random frequency (400 or $1000 \mathrm{~Hz}$ )

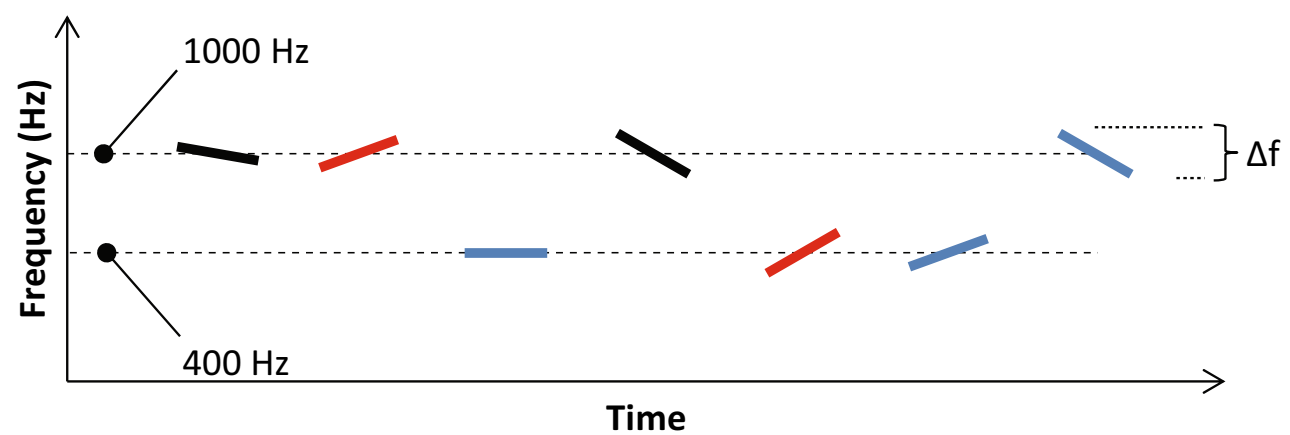

Fig. 1 (a) On each trial in Experiments 1 and 2, a linear frequency glide with a fixed center frequency of $1,000 \mathrm{~Hz}$ was presented. The frequency excursion $(\Delta \mathrm{f})$ varied randomly across trials over a range of preselected values and participants were asked to judge the direction of the glide (either upward or downward) by making an unspeeded response. (b) Experiment 3 was similar to Experiments 1 and 2 except that frequency glides centred around frequencies of 1,000 and $400 \mathrm{~Hz}$ were randomly interleaved. In all experiments, the data were binned into two categories

Procedure A trial started with the presentation of a central white fixation dot on a black screen for duration of $500 \mathrm{~ms}$. Subsequently, the linear frequency glide was presented for $100 \mathrm{~ms}$, and participants were required to judge its direction by either pressing the 0 or 1 key when the glide direction was downward or upward, respectively. The fixation dot disappeared when a response was made and the next trial was initiated after $200 \mathrm{~ms}$. The frequency excursion was randomly mixed over trials and balanced within blocks. Five experimental blocks were completed with 110 trials in each. Participants performed two sessions and each session was preceded by a practice block of 40 trials. The $\Delta \mathrm{fs}$ chosen for practice trials were $\pm 1 / 12, \pm 1 / 6, \pm 1 / 3$, and \pm 2 octaves so that participants were comfortable in performing the task. Before the experiment, participants received instructions on the screen informing them that the task was unspeeded and that accuracy was important. Participants were not given explicit instructions as to whether they should have their eyes open or closed, and chose whichever was most comfortable.

Analysis The intertrial adaptation paradigm involves a oneback analysis (Fig. 1a) of the glide direction responses to determine whether the PSC on a given trial is influenced by based on the preceding trial's glide direction and psychometric functions were fitted. One psychometric function was plotted for preceding upward glides (see the blue glides in panels a and $\mathbf{b}$ ), and another for preceding downward glides (see the red glides), with the function's mean indicating the point of subjective constancy (PSC). Trials in which the preceding auditory stimulus was constant in pitch were excluded (see black glides), as was the first trial of each block

exposure to the preceding trial's glide direction (see Van der Burg et al., 2013 for a similar methodology). Thus, separate distributions are plotted for trials in which the previous trial was a downward glide and for trials in which the previous trial was an upward glide. Any difference between the distributions can be attributed to the direction of the glide on trial $t-1$.

\section{Results and discussion}

To reveal the effect of inter-trial adaptation, distributions of perceived upward responses as a function of $\Delta \mathrm{f}$ were compiled for the case where trial $\mathrm{t}-1$ had a downward glide (negative $\Delta \mathrm{f}$ ) and for the case where trial $\mathrm{t}-1$ had an upward glide (positive $\Delta \mathrm{f}$ ). Each individual's distribution was fitted with a cumulative Gaussian distribution whose mean $(\mu)$ and standard deviation $(\sigma)$ were free to vary (Eq. 1). The mean of the best-fitting distribution is taken as the PSC.

$f(x)=0.5\left(1+\operatorname{erf}\left(\frac{x-\mu}{\sqrt{2 \sigma}}\right)\right)$ 
A two-tailed t-test was conducted on the PSC and standard deviation, with $\mathrm{t}-1$ glide direction as a within participant variable. The first trial of each block was excluded from analyses. The group mean responses as a function of $\Delta \mathrm{f}$ for the case where trial t-1 had a downward and for when trial t-1 had an upward glide along with the best-fitting cumulative Gaussian distribution are shown in Fig. 2. Separate two-tailed t-tests on mean PSC, $\mu$, and $\sigma$ were conducted to examine whether the distribution was affected by the preceding glide direction.

Figure 2 suggests that the direction of the glide in trial t-1 had an influence on performance in the predicted direction. The PSC was lower when the glide on the preceding trial was downward ( -0.005 octaves) than when the preceding glide was upward (.020 octaves). However, this effect on PSC was far from significant, $\mathrm{t}(7)=1.1, p=0.292$. There was no effect of the previous trial on the standard deviation, $\mathrm{t}(7)=1.7, p=$ 0.148 .

Although Fig. 2 points towards an intertrial adaptation effect, this was not statistically confirmed. Visual inspection of the individual psychometric functions reveals why: seven of eight participants showed a consistent adaptation effect in the expected direction (i.e., PSC following downward glides < PSC following upward glides, with excursions ranging from 0.02-0.12 octaves), whereas one participant showed a very large effect in the opposite direction (with a PSC difference of -0.11 octaves; Fig. 3a). When the data for the other seven participants are analysed (Fig. 3b), there is a clear and consistent adaptation effect, as predicted, which is confirmed by a two-tailed t-test $[\mathrm{t}(6)=3.4, p=0.015]$.

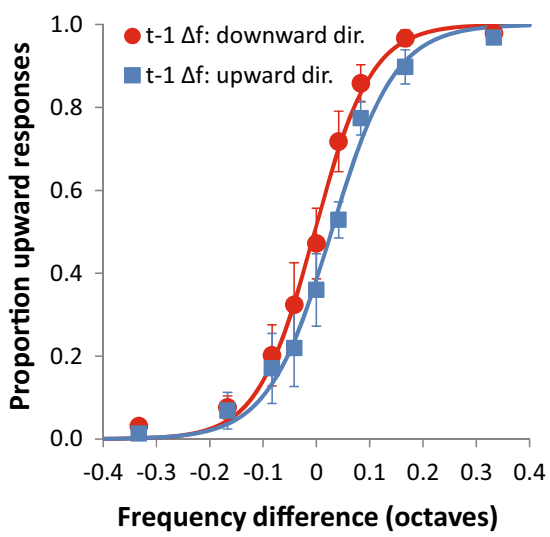

Fig. 2 Results of Experiment 1. The proportion of upward responses is plotted as a function of frequency excursion $(\Delta \mathrm{f})$. Negative $\Delta \mathrm{fs}$ indicate a downward glide, positive $\Delta \mathrm{fs}$ an upward glide. The data have been binned based on the glide direction in the previous trial $(\mathrm{t}-1)$ : blue squares indicate the preceding glide was upward, red circles indicate the preceding glide was downward. Continuous lines are best-fitting cumulative Gaussian distributions to the two t- 1 trial types. The PSC for each $\mathrm{t}-1$ trial type is given by the mean of the cumulative Gaussian. Note that the data points for $\Delta \mathrm{fs}$ of \pm 2 octaves are not shown in the figure but were included in the curve fitting. Group mean performance in the \pm 2 octave condition was very close to ceiling/floor, as expected ( 0.997 and 0.019 , respectively). All error bars represent \pm 1 SEM
The excluded participant's data show generally poor and biased performance on this task, as indicated by the broad slope of the psychometric functions (broader than the other seven participants) and a strong overall bias to perceive frequency sweeps as upward (PSCs are further to the left than the other participants). It is plausible that the participant adopted a top-down strategy to deal with their lack of acuity on this task, for example, simply repeating the response given on the preceding trial when the current direction is unclear. Rather than speculate on possible response biases or strategic responding, in Experiment 2 the experiment was repeated with a task that minimised top-down effects.

\section{Experiment 2}

The experiment was identical to Experiment 1, except that participants were asked to judge whether the pitch of the auditory stimulus changed or remained constant throughout the trial. The direction of the glide (whether an upwards or downwards change in pitch) is therefore task irrelevant as participants are simply detecting any change in pitch. This means a lateral shift in the response distribution in one direction cannot be attributed to systematic repetition (or alternation) of the preceding response (see also Spence \& Parise, 2010; Theeuwes \& Van der Burg, 2013; Van der Burg, Olivers, Bronkhorst, \& Theeuwes, 2008). As in Experiment 1, we predict that if adaptation to a linear frequency glide occurs rapidly based on a single trial, then the PSC on the current trial (t) should be contingent upon the direction of the frequency glide on the preceding trial ( $\mathrm{t}-1)$, even though the direction of the glide is task irrelevant. Specifically, the PSC should shift downward if the preceding glide was downward and upward if the preceding glide was upward.

\section{Method}

Eight students participated in the experiment ( 2 females; mean age: 25.1 years; range 21-29). All participants were naïve as to the purpose of the experiment. The experiment was identical to Experiment 1 except that participants' task was to judge whether the auditory stimulus changed in pitch or not (by pressing the 0 or $1 \mathrm{key}$, respectively) rather than by indicating the perceived direction of the glide. Importantly, the direction of the glide (up or down) is irrelevant to the task.

\section{Results and discussion}

To reveal the effect of intertrial adaptation, distributions of "perceived constant pitch" responses were compiled as a function of $\Delta \mathrm{f}$ for the case where trial t-1 had a downward 
Fig. 3 The proportion of upward responses as a function of frequency excursion $(\Delta \mathrm{f})$ for one deviant participant (panel a) and the remaining seven consistent participants (panel b) in Experiment 1. As in Fig. 2, blue squares indicate the preceding glide was upward, red circles indicate the preceding glide was downward and continuous lines are best-fitting cumulative Gaussian distributions. Error bars in panel b show \pm 1 SEM

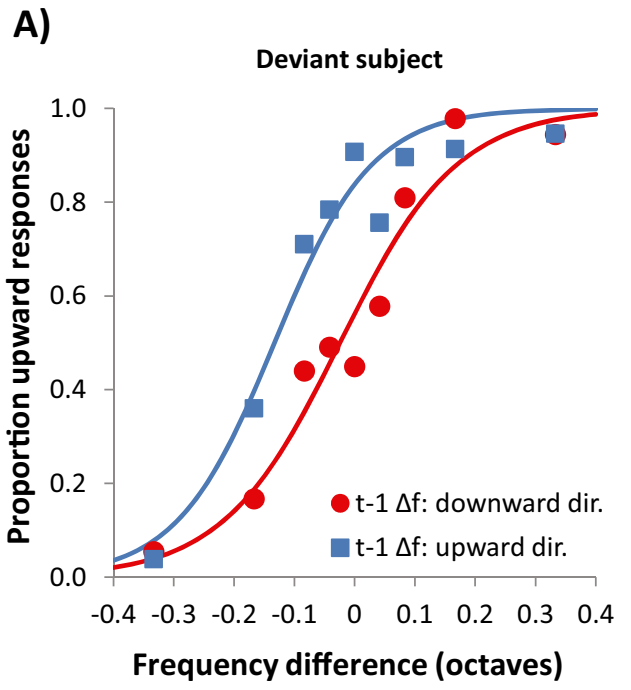

B)

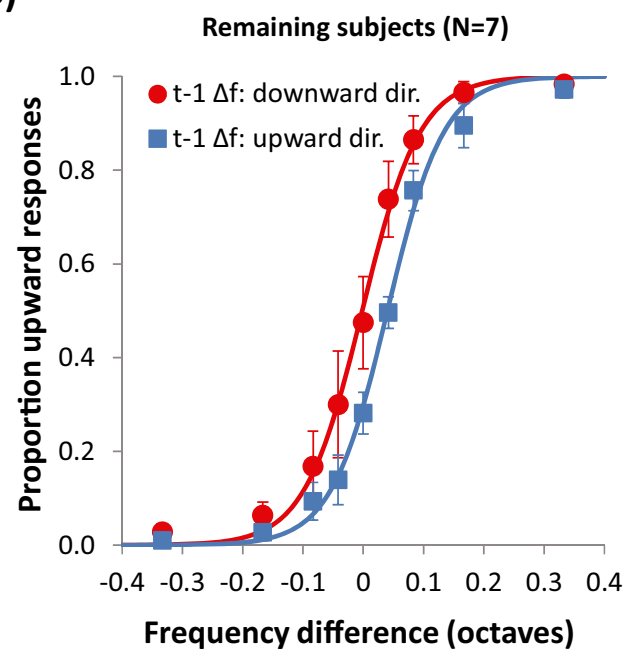

glide (negative $\Delta \mathrm{f}$ ) and for the case where trial t-1 had an upward glide (positive $\Delta \mathrm{f}$ ), as shown in Fig. 4 for the group mean data. The individual distributions were fitted with Gaussian normal distributions whose mean $(\mu)$, standard deviation $(\sigma)$ and amplitude (a) were free to vary (Eq. 2 ). The mean of the best-fitting distribution is taken as the PSC.

$f(x)=a e^{-(x-\mu)} \frac{-\left(x \sigma^{2}\right.}{2}$

Two-tailed $t$ tests were conducted on the PSC, standard deviation and amplitude parameters, with t-1 direction as a

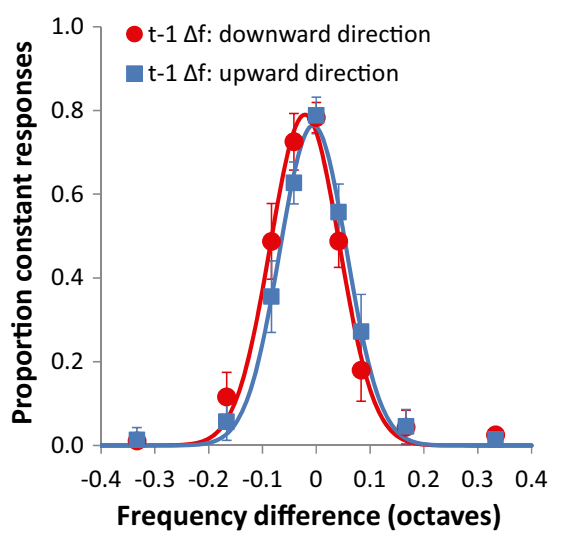

Fig. 4 Results of Experiment 2. The proportion of 'constant' responses as a function of frequency excursion $(\Delta \mathrm{f})$. Negative $\Delta \mathrm{fs}$ indicate a downward glide, positive $\Delta \mathrm{fs}$ an upward glide. The data have been binned based on the glide direction in the previous trial $(\mathrm{t}-1)$ : blue squares indicate the preceding glide was upward, red circles indicate the preceding glide was downward. Continuous lines are best-fitting Gaussian normal distributions to the two t- 1 trial types. The PSC for each t- 1 trial type is given by the mean of the Gaussian distribution. Note that the data points for $\Delta$ fs of \pm 2 octaves are not shown in the figure but were included in the curve fitting. Responses in the \pm 2 octave glide condition approached asymptotic performance (group mean of 0.004 ). All error bars represent \pm 1 SEM within participant variable. The first trial of each block was excluded from analyses.

There was a highly significant effect of $\mathrm{t}-1$ glide direction on PSC, $\mathrm{t}(7)=4.6, p=0.003$, with the PSC being lower when the preceding glide direction was downward (-0.022 octaves) than when it was upward $(-0.007$ octaves). The preceding glide direction had no effect whatsoever on the amplitude and standard deviation, $\mathrm{t}(7)=0.3, p=0.738$, and $\mathrm{t}(7)=1.6, p=$ 0.154 , respectively.

These results show clearly that although the direction of the glide was not relevant to the task, the PSC was still contingent upon the preceding trial's direction. The present experiment confirms that adaptation to linear frequency glides occurs rapidly from trial to trial without any explicit adaptation procedure and does so with a task that reduces the potential for response bias to influence the data.

An interesting question is whether the adaptation effect is entirely driven by the glide direction on the preceding trial or whether a longer history determines the adaptation strength. Therefore, we conducted an ANOVA on PSC with glide direction on $\mathrm{t}-1$ and glide direction on $\mathrm{t}-2$ as within-subject variables. Note that the first two trials of each block were discarded. The ANOVA on PSC yielded a highly significant effect of $\mathrm{t}-1$ glide direction on PSC, $\mathrm{F}(1,7)=20.3, p=0.003$, with the PSC being lower when the preceding glide direction was downward ( -0.023 octaves) than when the it was upward ( -0.005 octaves). There also was a significant effect on $\mathrm{t}-2$ glide direction on PSC, $\mathrm{F}(1,7)=9.4, p=0.018$, with the PSC being lower when the glide direction on trial $\mathrm{t}-2$ was downward $(-0.018$ octaves $)$ than when the it was upward ( -0.010 octaves). Interestingly, this $\mathrm{t}-2$ effect was independent of the glide direction on trial $t-1$, as the two-way interaction was far from significant, $\mathrm{F}(1,7)=2.0, p=$ 0.197 . This confirms that the glide direction on trial $\mathrm{t}-2$ was sufficient on its own to produce reliable recalibration effects. The $\mathrm{t}-2$ effect demonstrates that the state of adaptation arising for a 
given trial is not completely overwritten by a subsequent trial: a small but significant effect ( $\mathrm{t}-2$ effect of 0.008 octaves vs $\mathrm{t}-1$ effect of 0.018 octaves) will endure for at least one further trial.

\section{Experiment 3}

The purpose of Experiment 3 was to test whether the observed effect of rapid intertrial adaptation is frequency specific. We randomly interleaved two sets of linear frequency glides, one centered on 1,000 Hz (as in Experiments 1 and 2) and another on $400 \mathrm{~Hz}$. If intertrial adaptation to frequency glides is frequency specific, then we expect to replicate the adaptation effect when consecutive trials are drawn from the same frequency (e.g., $400 \mathrm{~Hz}$ in trial t-1, and $400 \mathrm{~Hz}$ in trial t) but not when consecutive trials are drawn from different sets (e.g., $400 \mathrm{~Hz}$ in trial $\mathrm{t}-1$, and $1000 \mathrm{~Hz}$ in trial t).

\section{Method}

Eight students participated in the experiment (4 females; mean age: 23.4 years; range 20-27). All participants were naïve as to the purpose of the experiment. The experiment used the "tone constant" task from Experiment 2 and was identical to it except for the following points. On half of the trials the linear frequency glide had a center frequency of $1,000 \mathrm{~Hz}$ (as in Experiments 1 and 2), and on the remaining trials the center frequency was $400 \mathrm{~Hz}$ (Fig. 1b). For each center frequency there was a set of glides defined by $\Delta \mathrm{fs}$ of $(0, \pm 1 / 24, \pm 1 / 12$, $\pm 1 / 6, \pm 1 / 3$, and \pm 1 octaves). The center frequencies (400 and $1,000 \mathrm{~Hz}$ ) and $\Delta \mathrm{fs}$ were presented in a completely random order and balanced within blocks (corresponding to glides sweeping between 986-1015, 972-1029, 944-1060, 8911123, and 707-1414 Hz and 394-406, 389-412, 378-424, 356-449, and 282-567 Hz). Ten experimental blocks were completed with 110 trials in each. Participants performed two sessions, and each session was preceded by a practice block of 40 trials. The data were analysed as in Experiment 2 by calculating for every participant the mean proportion of "constant" responses in each glide level and fitting a Gaussian distribution to the responses to determine the PSC, standard deviation, and amplitude.

\section{Results and discussion}

The group mean results of Experiment 3 are illustrated in Fig. 5. Results are grouped based on whether consecutive trials had different center frequencies $(400 \mathrm{~Hz}$ followed by $1,000 \mathrm{~Hz}$, or $1,000 \mathrm{~Hz}$ followed by $400 \mathrm{~Hz}$ ) or the same center frequency (both $400 \mathrm{~Hz}$ or both $1,000 \mathrm{~Hz}$ ) after an initial analysis showed the same/different effects did not differ with frequency. In panel a, it is clear there is no difference between the distributions for preceding upward glides and preceding downward glides when consecutive trials have different center frequencies. In contrast, when consecutive trials have the same center frequency (panel b), the two curves replicate the effect reported in Experiment 2, with a lower PSC following downward glides and a higher PSC following upward glides. To confirm this, we conducted an ANOVA on the PSC, standard deviation and amplitude parameters with t-1 frequency excursion and intertrial frequency (same vs. different) as within participant variables. Panel $\mathrm{c}$ shows the group mean of the best-fitting parameters across the eight participants.

The ANOVA on PSC yielded a reliable two-way interaction between preceding glide direction and inter-trial frequency, $\mathrm{F}(1,7)=15.5, p=0.006$ (Fig. $5 \mathrm{c}$, bottom panel). This interaction was further examined by two-tailed $t$ tests. Interestingly, the preceding glide direction had an effect on the PSC when the frequency in trial $t$ was the same as in trial $t-1$, $\mathrm{t}(7)=4.1, p=0.005$, as the PSC was significantly lower when the preceding glide direction was downward ( -0.040 octaves) than when it was upward ( -0.018 octaves). As is clear from Fig. 5a, no such PSC shift was observed when the center frequency differed between trial $\mathrm{t}$ and trial $\mathrm{t}-1, \mathrm{t}(7)=0.2, p=$ 0.856 . The main effects of inter-trial frequency and $\mathrm{t}-1$ glide direction were not reliable $[\mathrm{F}(1,7)=3.5, p=0.102$, and $\mathrm{F}(1$, 7) $=4.2, p=0.080$, respectively].

The ANOVA on standard deviation yielded no main effect of inter-trial frequency, $\mathrm{F}(1,7)=3.7, p=0.095$, nor $\mathrm{t}-1$ direction, $\mathrm{F}(1,7)=0.1, p=0.719$, and neither was their interaction reliable, $\mathrm{F}(1,7)=2.4, p=0.162$.

The ANOVA on amplitude yielded a reliable two-way interaction between preceding glide direction and inter-trial frequency, $\mathrm{F}(1,7)=7.0, p=0.033$ (Fig. $5 \mathrm{c}$, top panel). This interaction was further examined by two-tailed $t$ tests. The preceding glide direction had an effect on amplitude when the frequency in trial $\mathrm{t}$ was the same as in trial $\mathrm{t}-1, \mathrm{t}(7)=3.6, p=$ 0.009 , as the amplitude was significantly lower when the glide direction in trial t-1 was upward (0.71) than when it was downward (0.81). No effect on amplitude was observed when the center frequency differed between trial $\mathrm{t}$ and trial $\mathrm{t}-1, \mathrm{t}(7)=$ $0.8, p=0.467$. The main effect of intertrial frequency was not significant, $\mathrm{F}(1,7)=1.6, p=0.250$, although the main effect of $\mathrm{t}-1$ glide direction was significant, $\mathrm{F}(1,7)=7.3, p=0.030$.

\section{Discussion}

In this study, we have shown that adaptation to frequency modulation occurs extremely rapidly, being present after exposure to a single frequency sweep of $100 \mathrm{~ms}$ duration. This is demonstrated by shifts in the PSC dependent on the frequency glide direction in the previous trial $(\mathrm{t}-1)$. Although adaptation for frequency modulation has been reported previously 
A)

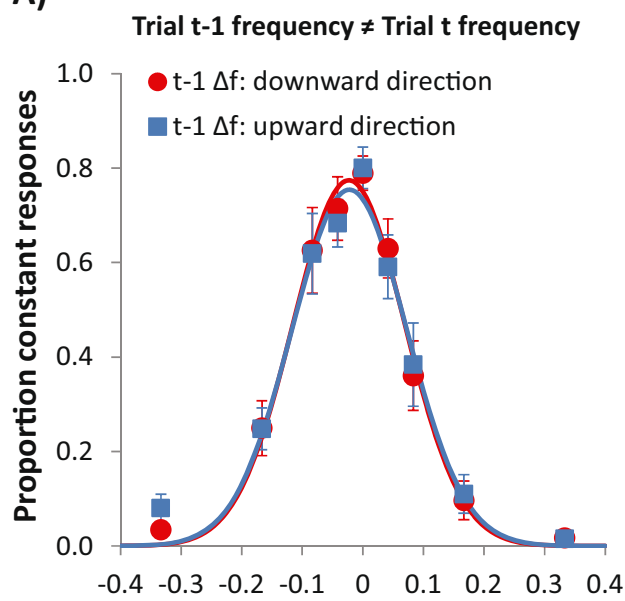

Frequency difference (octaves)
B)

Trial $t-1$ frequency $=$ Trial $t$ frequency

- $\mathrm{t}-1 \Delta \mathrm{f}$ : downward direction

t-1 $\Delta \mathrm{f}$ : upward direction

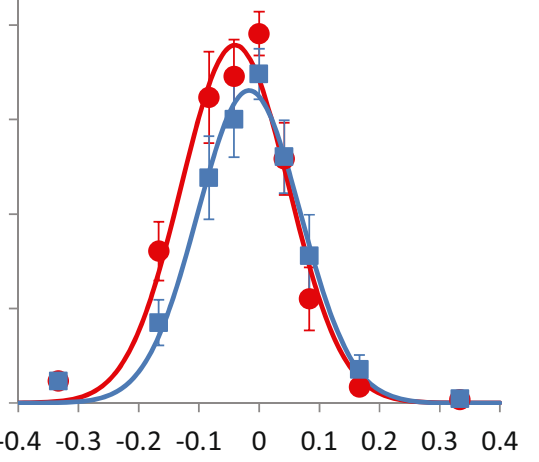

Frequency difference (octaves)
C)

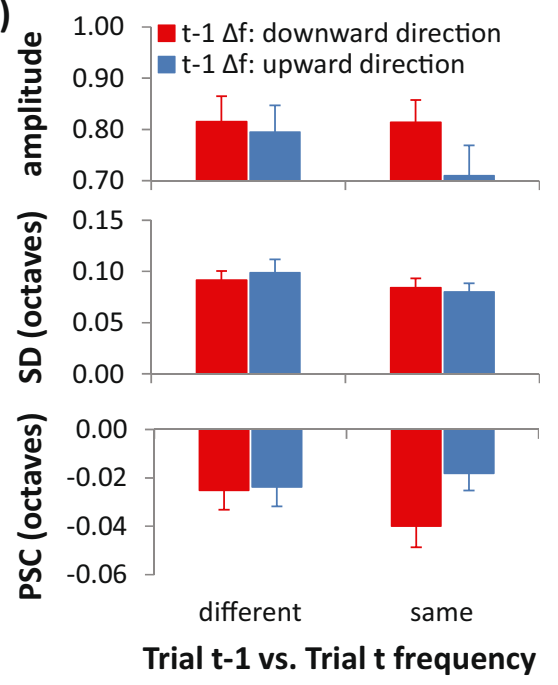

Fig. 5 Results of Experiment 3. Panels a and b show the proportion of "constant" responses as function of frequency excursion $(\Delta f)$. Negative $\Delta$ fs indicate a downward glide, positive $\Delta$ fs an upward glide. The data have been binned based on the glide direction in the previous trial (t-1): blue squares indicate the preceding glide was upward, red circles indicate the preceding glide was downward. Continuous lines are best-fitting Gaussian normal distributions to the two t- 1 trial types. The PSC for each t-1 trial type is given by the mean of the Gaussian distribution. Note that the data points for $\Delta \mathrm{fs}$ of \pm 1 octave are not shown in the figure but

(Gardner \& Wilson, 1979; Green \& Kay, 1973; Kay \& Matthews, 1972; Kayahara, 2001; Masutomi \& Kashino, 2013; Regan \& Tansley, 1979; Shu et al., 1993; Tansley \& Suffield, 1983), here we find that it can occur rapidly and without an explicit adaptation procedure (Experiments 2 and 3). In addition to significant shifts resulting from the previous glide, the perception of glide direction is also independently influenced by the $\mathrm{t}-2$ glide. Experiment 3 shows that these rapid adaptive shifts in PSC do not occur when the frequency of consecutive stimuli differ substantially, indicating the rapid adaptation effect is frequency tuned. In addition to these findings, we find when participants are required to judge the direction of frequency glide, a range of factors which may include topdown strategies or poor acuity may obscure the adaptation effect (Experiment 1), although this can be avoided using a task in which change detection rather than discrimination is used (Experiments 2 and 3).

Previous studies of frequency-modulation adaptation have shown the aftereffect to be direction-specific. Exposure to upward frequency glides alters detection of upward frequency change but not downward frequency change, and vice versa (Gardner \& Wilson, 1979; Kayahara, 2001). Our findings are consistent with these observations as we showed that upward frequency glides in the previous trial shifted the PSC higher so that an upward glide was more likely to be perceived as having constant pitch, and vice versa. Previous research has also shown the frequency-modulation aftereffect has a broad tuning to the carrier frequency (Kay \& Matthews, 1972; were included in the curve fitting. Responses in the \pm 1 octave glide condition approached asymptotic performance (group mean 0.009). Panel a shows intertrial data for the case where consecutive trials had different center frequencies ( $400 \mathrm{~Hz}$ followed by $1,000 \mathrm{~Hz}$, or vice versa), and panel $b$ shows intertrial data for the case where consecutive trials had the same center frequency (both $400 \mathrm{~Hz}$ or both 1,000 Hz). C) Group mean amplitude, standard deviation, and PSC as a function of preceding glide direction and inter-trial frequency. All error bars represent \pm 1 SEM

Kayahara, 2001; Okada \& Kashino, 2003), this is also true for our rapid intertrial adaptation effect. The similarities between the aftereffects produced by prolonged adaptation and intertrial adaptation paradigms suggest they reflect changes in the same underlying mechanism. The common interpretation in previous studies of frequency-modulation aftereffects is that a subset of FM-selective neurons tuned to the frequency modulation of the adaptor has their response properties altered, producing a population imbalance that manifests as the aftereffect. Most studies of FM aftereffects have adopted a version of this sensory adaptation account (Gardner \& Wilson, 1979; Green \& Kay, 1973; (Kay \& Matthews, 1972; Kayahara, 2001; Okada \& Kashino, 2003; Masutomi \& Kashino, 2013; Regan \& Tansley, 1979; Shu et al., 1993; Tansley \& Suffield, 1983). Other research, however, has questioned whether frequency modulation is encoded as a primary feature of sounds that is detected by dedicated FMselective neurons (Sek \& Moore, 1999; Lyzenga, Carlyon, \& Moore, 2004; Demany, Carlyon, \& Semal, 2009). These papers have argued that detection and discrimination of frequency glides may be accomplished by the same mechanisms that detect discrete frequency changes, especially when glides are near threshold. For glides that are well above threshold, as is clearly the case for our stimuli (only the smallest glide of $1 / 24$ th octave was near threshold), it has been argued that the auditory system can encode FM as a primary sound feature (Cusack \& Carlyon, 2003; Carlyon et al., 2004). In either case, frequency glides would be encoded by auditory mechanisms 
responsive to features of the stimuli and thus provide a basis for adaptation and aftereffects.

Although we favour the interpretation, in line with many previous papers, that the FM aftereffects reported here are due to sensory adaptation, two notable exceptions to this consensus suggest that FM aftereffects may be due to shifts in decision criteria (Moody et al., 1984; Wakefield \& Viemeister, 1984). Moody et al. (1984) demonstrated that differences in FM thresholds between adapted and nonadapted conditions decreased over repeated experimental sessions and proposed that this was more consistent with nonsensory factors such as criterion shifts than with sensory adaptation. A similar suggestion was made by Wakefield and Viemeister (1984) who proposed FM aftereffects were the result of comparison of the current trial with a reference that had been distorted by exposure to the adaptor. Neither of these studies establishes definitively that FM aftereffects are due to decision-level effects, and in any case our approach differs critically from these studies in that it was not a conventional aftereffect study. That is, there was no prolonged adaptation phase in our study with a repeating adaptor stimulus, limiting the scope for a distorted reference to develop. Instead, our study involved a series of randomly varying frequency sweeps and thus there was no explicit adaptor. Even if the set of trials preceding a given trial is taken collectively to be the adaptor, the mean of the preceding frequency sweeps is zero. Another argument against our results being due to decision-level effects (discussed further below) is that there was a significant effect on the current trial from the $\mathrm{t}-2$ trial, an effect that was independent of the $\mathrm{t}-1$ trial.

A number of previous studies, particularly using temporal judgements (Spence \& Parise, 2010; Theeuwes \& Van der Burg, 2013; Van der Burg et al., 2008), have drawn attention to the possible confounding influence of top-down strategies in tasks where discrimination is used (e.g., of temporal order: earlier vs. later) rather than detection (e.g., of simultaneity: simultaneous or not). The present study supports this suggestion as the glide direction discrimination task produced a highly deviant pattern of data in one participant in Experiment 1. Conceivably, this participant made responses based on the glide direction indicated in the previous trial, especially when the $\Delta f$ was small and the glide direction ambiguous. This participant's responding strategy produced a large effect in the opposite direction to all other observers. Importantly, an arbitrary response strategy of another kind could potentially exaggerate the effect in the expected direction. Either way, these strategic influences are best avoided and our results show that the constancy task used in Experiments 2 and 3 is more appropriate. When the direction of frequency change is task irrelevant, response bias is minimised and the very consistent data in Experiments 2 and 3 attest to this. Still, response bias is difficult to eliminate entirely and the question remains: could response bias explain our intertrial adaptation effects, even when using the constancy task?

The use in Experiment 3 of interleaved sets of frequency sweeps based on different centre frequencies was designed to test whether our results were due to sensory adaptation or decision-level factors. We reasoned that if the PSC shifts found here were due to sensory adaptation in FM-tuned neurons that it would exhibit frequency specificity, whereas strategic responding based on the direction of the previous sweep would not be frequency specific and we would obtain the same pattern of results when consecutive trials shared the same center frequency as when they did not. The data in Fig. 5 show there was no intertrial adaptation effect when consecutive frequencies differed, while a reliable effect occurred when consecutive frequencies were the same. This is consistent with the prediction of the sensory adaptation effect, although it does not necessarily rule out a decisional account as it may be argued that shifts in decision criteria occur only when the centre frequency remains the same. One theory that provides an explanation for trial-by-trial alterations in decision criterion settings is criterion-setting theory (CST) (Treisman \& Williams, 1984; Treisman et al., 1995; Lages \& Treisman, 2010). CST is an extension of signal detection theory, proposing that response criteria vary from trial-to-trial with the goal of optimising responses to the current stimulus. The criterion is adjusted based on the sum of a positive indicator trace (stabilization mechanism) and a negative indicator trace (probability tracking mechanism), which are differentially weighted. Whether a criterion-shifting model could account for our data is unclear as studies have most commonly reported positive sequential dependencies (Treisman \& Williams, 1984; Treisman et al., 1995), whereas our data show negative dependencies. The results of our $\mathrm{t}-2$ analysis are relevant here. The significant effect of trial $t-2$ on the current trial's response, independent of the sweep direction in trial $t-1$, provides evidence against a decisional account, because it shows that responding based on the previous trial's direction cannot account for the data. In another recent study of sequential dependencies (Fischer \& Whitney, 2014), subjects were tested explicitly on their recall of the $\mathrm{t}-2$ stimulus and performed at chance level on this task even though $\mathrm{t}-2$ trials still showed a significant serial effect, a finding which adds weight to lowerlevel sensory accounts of sequential dependencies.

Although CST provides a framework for explaining sequential dependencies using criterion changes, two notable recent papers have proposed that sequential dependencies may reflect sensory changes (Chopin \& Mamassian, 2012; Fischer \& Whitney, 2014). Fischer and Whitney (2014) described a series of experiments demonstrating that the perceived orientation of a briefly presented Gabor patch depended on what orientation had been seen in the previous trial. Unlike our study, their intertrial effect was an attraction, 
with the current orientation attracted towards that of the previously viewed Gabor. This is similar to other work on sequential dependence showing repetition priming of attentional shifts, which serves to optimise responses to features or locations that have been relevant in the recent past (Kristjansson, 2008; Kristjansson, \& Campana, 2010; Sigurdardottir et al., 2008). It should be noted that although the sequential dependencies for orientation reported by Fischer and Whitney were attractive for brief presentations, repulsive effects occurred when the presentation duration was prolonged. This differs from FM aftereffects, which are in the same direction following both brief and prolonged presentations, as are spatial and temporal recalibration across vision and audition (Van der Burg et al., 2013; Wozny \& Shams, 2011). Our sensory systems may balance attraction effects such as priming and positive sequential dependencies, which exploit continuity, against the repulsive effects of adaptation, which enhances sensitivity to change (see Fischer \& Whitney, 2014).

In cross-modal research (Alais, Newell, \& Mamassian, 2010), previous studies using the intertrial adaptation paradigm with audiovisual stimuli have shown the aftereffects are comparable in magnitude to those obtained with prolonged exposure paradigms (Harvey et al., 2014; Van der Burg et al., 2013, 2014; Wozny \& Shams, 2011) and that the effects are even present and strong when participants passively perceive the audiovisual stimuli (Van der Burg et al. 2013). Although an early report suggested that the magnitude plateaus after 1220 s of adaptation (Kay \& Matthews, 1972), two subsequent studies investigating the time-course of the aftereffect concluded that the aftereffect accrues rapidly in the first few minutes but does not saturate until 20-30 minutes (Regan \& Tansley, 1979; Tansley \& Suffield, 1983). In this study, we find that reliable changes can be demonstrated after a single trial lasting just $100 \mathrm{~ms}$. The study most directly comparable to our rapid adaptation approach presented a brief adaptor stimulus six times prior to each test stimulus, with both adapting and test stimuli consisting of linear frequency glides centered on $1000 \mathrm{~Hz}$ (Masutomi \& Kashino, 2013). This paradigm produced an aftereffect magnitude of about 0.05 octaves, larger than the aftereffects found in our study of 0.015 and 0.022 octaves, for Experiments 2 and 3, respectively. In addition to the duration of exposure, a number of methodological differences make the cause of the magnitude difference unclear. In particular, the stimuli were of much longer duration $(800 \mathrm{~ms}$ in their study compared with $100 \mathrm{~ms}$ here) and a judgement of glide direction (rather than constancy) was used. These factors may have contributed to the difference in magnitude, especially their longer adaptation period. However, regardless of these differences, the statistically reliable adaptation effects we report here demonstrate the utility of the intertrial methodology and highlight that effective frequency modulation adaptors can be very brief indeed.
The results reported here show that adaptation to frequency modulation occurs extremely rapidly, without requiring prolonged exposure to adapting stimuli. Indeed, our data show that reliable PSC shifts occur after exposure to a single trial of just $100 \mathrm{~ms}$. Furthermore, the effect of the direction of an auditory glide on participant's responses persists over two trials and is independent of the intervening trial. The shift in PSC is frequency tuned and most robust using a constancy task, which helps minimise any role for response bias and topdown strategies. The intertrial adaptation paradigm, therefore, provides an efficient approach to investigating aftereffects within a single modality, as has been shown in recent studies of multisensory adaptation (Van der Burg et al., 2013; Wozny \& Shams, 2011, but see Harvey et al., 2014). The fact that reliable PSC shifts occur after only $100 \mathrm{~ms}$ of adaptation implies that the mechanisms encoding frequency modulation are constantly adapting to their inputs, however brief, and are continually involved in an adaptive process of "tuning in" to the statistical properties of the auditory signals generated in our environment.

Acknowledgments This research was supported by grants from the Australian Research Council to D. Alais (Discovery Project, DP120101474), and to E. Van der Burg (Discovery Early Career Research Award, DE130101663).

\section{References}

Alais, D., Newell, F. N., \& Mamassian, P. (2010). Multisensory processing in review: From physiology to behaviour. Seeing and Perceiving, 23(1), 3-38.

Altmann, C. F., \& Gaese, B. H. (2014). Representation of frequencymodulated sounds in the human brain. Hearing Research, 307, 74-85.

Atencio, C. A., Blake, D. T., Strata, F., Cheung, S. W., Merzenich, M. M., \& Schreiner, C. E. (2007). Frequency-modulation encoding in the primary auditory cortex of the awake owl monkey. Journal of Neurophysiology, 98(4), 2182-2195.

Carlile, S., Hyams, S., \& Delaney, S. (2001). Systematic distortions of auditory space perception following prolonged exposure to broadband noise. The Journal of the Acoustical Society of America, $110(1), 416$.

Carlyon, R. P., Micheyl, C., Deeks, J. M., \& Moore, B. C. J. (2004). Auditory processing of real and illusory changes in frequency modulation (FM) phase. The Journal of the Acoustical Society of America, 116(6), 3629-3639.

Chopin, A., \& Mamassian, P. (2012). Predictive properties of visual adaptation. Current Biology, 22, 622-626.

Clifford, C. W. G., Webster, M. A., Stanley, G. B., Stocker, A. A., Kohn, A., Sharpee, T. O., \& Schwartz, O. (2007). Visual adaptation: neural, psychological and computational aspects. Vision Research, 47(25), $3125-3131$.

Cusack, R., \& Carlyon, R. P. (2003). Perceptual asymmetries in audition. Journal of Experimental Psychology: Human Perception and Performance, 29(3), 713-725.

Dalton, P. (2000). Psychophysical and Behavioral Characteristics of Olfactory Adaptation. Chemical Senses, 25(4), 487-492. 
Delattre, P., Cooper, F. S., Liberman, A. M., \& Gerstman, L. (1954). Acoustic Loci and transitional cues for consonants. The Journal of the Acoustical Society of America, 26, 137.

Demany, L., Carlyon, R. P., \& Semal, C. (2009). Continuous versus discrete frequency changes: Different detection mechanisms? The Journal of the Acoustical Society of America, 125(2), 1082-1090.

Fisher, J., \& Whitney, D. (2014). Serial dependence in visual perception. Nature Neuroscience, 17(5), 738-743.

Frisby, J. P. (1980). Seeing: illusion, brain, and mind. New York: Oxford University Press.

Gardner, R. B., \& Wilson, J. P. (1979). Evidence for direction-specific channels in the processing of frequency modulation. The Journal of the Acoustical Society of America, 66(3), 704-709.

Gordon, M., \& O'Neill, W. E. (1998). Temporal processing across frequency channels by FM selective auditory neurons can account for FM rate selectivity. Hearing Research, 122(1-2), 97-108.

Green, G. G., \& Kay, R. H. (1973). Proceedings: The adequate stimuli for channels in the human auditory pathways concerned with the modulation present in frequency-modulated tones. The Journal of Physiology, 234(2), 50P-52P.

Hahn, J. F. (1966). Vibrotactile adaptation and recovery measured by two methods. Journal of Experimental Psychology, 71(5), 655-658.

Harvey, C., Van der Burg, E., \& Alais, D. (2014). Rapid recalibration occurs crossmodally without stimulus specificity but is absent unimodally. Brain Research. doi:10.1016/j.brainres.2014.08.028

Hollins, M., Sliman, J. B., \& Washburn, S. (2001). Vibrotactile adaptation impairs discrimination of fine, but not coarse, textures. Somatosensory and Motor Research, 18(4), 253-262.

Kay, R. H., \& Matthews, D. R. (1972). On the existence in human auditory pathways of channels selectively tuned to the modulation present in frequency-modulated tones. The Journal of Physiology, 225(3), 657-677.

Kayahara, T. (2001). Aftereffect of adaptation to uni-directional frequency change: Evidence for selective processing mechanism. Acoustical Science and Technology, 22(1), 49-51.

Kristjansson, A. (2008). "I know what you did on the last trial-"a selective review of research on priming in visual search. Frontiers in Bioscience, 13, 1171-1181.

Kristjansson, A., \& Campana, G. (2010). Where perception meets memory: A review of repetition priming in visual search tasks. Attention, Perception \& Psychophysics, 72(1), 5-18.

Lages, M., \& Treisman, M. (2010). A criterion setting theory of discrimination learning that accounts for anisotropies and context effects. Seeing and Perceiving, 23(5-6), 401-434.

Liang, L., Lu, T., \& Wang, X. (2002). Neural representations of sinusoidal amplitude and frequency modulations in the primary auditory cortex of awake primates. Journal of Neurophysiology, 87(5), 2237-2261.

Liberman, A. M., \& Mattingly, I. G. (1989). A specialization for speech perception. Science, 243(4890), 489-494.

Liberman, A. M., Cooper, F. S., Shankweiler, D. P., \& Studdert-Kennedy, M. (1967). Perception of the speech code. Psychological Review, 74(6), 431-461.

Lyzenga, J., Carlyon, R. P., \& Moore, B. C. J. (2004). The effects of real and illusory glides on pure-tone frequency discrimination. The Journal of the Acoustical Society of America, 116(1), 491-501.

Masutomi, K., \& Kashino, M. (2013). Frequency-change aftereffect produced by adaptation to real and illusory unidirectional frequency sweeps. The Journal of the Acoustical Society of America, 134(1), EL14.

Mendelson, J. R., \& Cynader, M. S. (1985). Sensitivity of cat primary auditory cortex (Al) neurons to the direction and rate of frequency modulation. Brain Research, 327(1-2), 331-335.

Mendelson, J. R., \& Grasse, K. L. (1992). A comparison of monaural and binaural responses to frequency modulated (FM) sweeps in cat primary auditory cortex. Experimental Brain Research, 91(3), 435-454.

Mendelson, J. R., Schreiner, C. E., Sutter, M. L., \& Grasse, K. L. (1993). Functional topography of cat primary auditory cortex: Responses to frequency-modulated sweeps. Experimental Brain Research, 94(1), 65-87.

Miyazaki, M., Yamamoto, S., Uchida, S., \& Kitazawa, S. (2006). Bayesian calibration of simultaneity in tactile temporal order judgment. Nature Neuroscience, 9(7), 875-877.

Moody, D. B., Cole, D., Davidson, L. M., \& Stebbins, W. C. (1984). Evidence for a reappraisal of the psychophysical selective adaptation paradigm. The Journal of the Acoustical Society of America, 76(4), 1076-1079.

Okada, M., \& Kashino, M. (2003). The role of spectral change detectors in temporal order judgment of tones. NeuroReport, 14(2), 261-264.

Pardo, P. J., \& Sams, M. (1993). Human auditory cortex responses to rising versus falling glides. Neuroscience Letters, 159(1-2), 43-45.

Phillips, D. P., \& Hall, S. E. (2005). Psychophysical evidence for adaptation of central auditory processors for interaural differences in time and level. Hearing Research, 202(1-2), 188-199.

Pryor, G. T., Steinmetz, G., \& Stone, H. (1970). Changes in absolute detection threshold and in subjective intensity of suprathreshold stimuli during olfactory adaptation and recovery. Perception \& Psychophysics, 8(5), 331-335.

Regan, D., \& Tansley, B. W. (1979). Selective adaptation to frequencymodulated tones: Evidence for an information-processing channel selectively sensitive to frequency changes. The Journal of the Acoustical Society of America, 65(5), 1249-1257.

Sams, M., Aulanko, R., Hämäläinen, M., Hari, R., Lounasmaa, O. V., Lu, S. T., \& Simola, J. (1991). Seeing speech: Visual information from lip movements modifies activity in the human auditory cortex. Neuroscience Letters, 127(1), 141-145.

Sek, A., \& Moore, B. C. (1999). Discrimination of frequency steps linked by glides of various durations. The Journal of the Acoustical Society of America, 106(1), 351-359.

Shu, Z. J., Swindale, N. V., \& Cynader, M. S. (1993). Spectral motion produces an auditory after-effect. Nature, 364(6439), 721-723.

Sigurdardottir, H. M., Kristjansson, A., \& Driver, J. (2008). Repetition streaks increase perceptual sensitivity in visual search of brief displays. Visual Cognition, 16(5), 643-658.

Spence, C., \& Parise, C. (2010). Prior-entry: A review. Consciousness and Cognition, 19(1), 364-379.

Steinmetz, G., Pryor, G. T., \& Stone, H. (1970). Olfactory adaptation and recovery in man as measured by two psychophysical techniques. Perception \& Psychophysics, 8(5), 327-330.

Tansley, B. W., \& Suffield, J. B. (1983). Time course of adaptation and recovery of channels selectively sensitive to frequency and amplitude modulation. The Journal of the Acoustical Society of America, 74(3), 765-775.

Theeuwes, J., \& Van der Burg, E. (2013). Priming makes a stimulus more salient. Journal of Vision, 13(3), 1-11.

Tian, B., \& Rauschecker, J. P. (2004). Processing of frequency-modulated sounds in the lateral auditory belt cortex of the rhesus monkey. Journal of Neurophysiology, 92(5), 2993-3013.

Treisman, M., \& Williams, T. C. (1984). A theory of criterion setting with an application to sequential dependencies. Psychological Review, 91(1), 68-111.

Treisman, M. A., Faulkner, A., Naish P. L. N., \& Rosner, B. S. (1995). Voice-onset time and tone-onset time: The role of criterion-setting mechanisms in categorical perception. Quarterly Journal of Experimental Psychology, 48, 334-366.

Van der Burg, E., Olivers, C. N. L., Bronkhorst, A. W., \& Theeuwes, J. (2008). Audiovisual events capture attention: Evidence from temporal order judgments. Journal of Vision, 8(5), 2.1-10. 
Van der Burg, E., Alais, D., \& Cass, J. (2013). Rapid recalibration to audiovisual asynchrony. The Journal of Neuroscience, 33(37), 14633-14637.

Van der Burg, E., Orchard-Mills, E., \& Alais, D. (2014). Rapid temporal recalibration is unique to audiovisual stimuli. Experimental Brain Research. doi:10.1007/s00221-014-4085-8

Wakefield, G. H., \& Viemeister, N. F. (1984). Selective adaptation to linear frequency-modulated sweeps: Evidence for direction-specific FM channels? The Journal of the Acoustical Society of America, 75(5), 1588-1592.

Webster, M. A. (2011). Adaptation and visual coding. Journal of Vision, 11(5), 1-23.
Wozny, D. R., \& Shams, L. (2011). Recalibration of auditory space following milliseconds of cross-modal discrepancy. The Journal of Neuroscience, 31(12), 4607-4612.

Zeng, F.-G., Nie, K., Stickney, G. S., Kong, Y.-Y., Vongphoe, M., Bhargave, A., Wei, C., \& Cao, K. (2005). Speech recognition with amplitude and frequency modulations. Proceedings of the National Academy of Sciences of the United States of America, 102(7), 22932298.

Zhao, H. B., \& Liang, Z. A. (1996). Processing of modulation frequency in the dorsal cochlear nucleus of the guinea pig: Sinusoidal frequency-modulated tones. Hearing Research, 95(1-2), $120-134$. 